\title{
Fijación quirúrgica de fracturas costales con placas de titanio: reporte de dos casos
}

\author{
MAURICIO FICA D.*, PATRICIO FERNÁNDEZ A.*, FRANCISCO SUÁREZ V.*, \\ RODRIGO APARICIO R.* y CLAUDIO SUÁREZ C.*
}

\section{Surgical repair of rib fractures using titanium plates: Report of two cases}

Rib fractures are common lesions in blunt chest trauma. Disregarding the severity of other high energy associated lesions, chest wall trauma characteristically causes intense pain, respiratory complications and long-term disability. Pain relief and chest wall function restoration are obtained by surgical stabilization of rib fractures. In nowdays still there is a considerable variability in surgical techniques and devices, as well as in their results and clinical indications. We report two cases of chest wall trauma and rib fractures repaired with osteosynthesis (Synthes ${ }^{\circledR}$ system MatrixRIB. Solothurn, Switzerland) and we discuss their new clinical indications and results.

Key words: Thoracic trauma; rib fractures; osteosynthesis.

\section{Resumen}

Las fracturas costales son lesiones frecuentes en los traumatismos torácicos contusos. Sin contar aquellas lesiones asociadas con la alta absorción de energía, las fracturas costales causan característicamente dolor intenso, complicaciones respiratorias y ausentismo laboral significativo. Una de las estrategias terapéuticas es la estabilización quirúrgica del foco de fractura, la cual tiene múltiples ventajas teóricas como la disminución del dolor y la restauración de la función de la pared costal. Existen múltiples formas de fijación costal, pero su indicación, técnica quirúrgica y resultados son muy disímiles. Presentamos dos casos de reparación de pared torácica con material de osteosíntesis especialmente diseñado para este efecto (Synthes ${ }^{\circledR}$ MatrixRIB, Solothurn, Suiza), y se discuten sus alcances en las indicaciones y resultados clínicos.

Palabras clave: Trauma torácico; fracturas costales; osteosíntesis.

\section{Introducción}

Las fracturas costales son lesiones que frecuentemente acompañan a los traumatismos torácicos y en especial si requieren de hospitalización. Por sí solas predicen morbilidad y mortalidad dado que son indicadores de la energía absorbida. Sirmali y cols ${ }^{1}$ en una serie de 548 hospitalizaciones por trauma torácico con fracturas costales $(39 \%$ de la admisiones por traumatismo torácico), muestra que el $72 \%$ de ellos presentó hemotórax, neumotórax o una combinación de ellos, y el número de muertes, lesiones asociadas y morbilidad se correlacionó con el número de fracturas costales .

Sin considerar la gravedad de las lesiones asociadas, las fracturas costales se caracterizan por producir gran dolor, morbilidad respiratoria y ausentismo laboral. Según Kerr-Valentic y cols ${ }^{2}$ un número significativo de pacientes presenta pasados los 30 días del trauma, dolor y alteración en prácticamente todos los dominios del cuestionario de calidad de vida SF-36, y además tardan más de 50 días en su reintegro laboral o nivel de actividad diaria habitual.

El manejo de las fracturas costales es clásicamente no intervencional, aún en situaciones con gran destrucción de la pared torácica, como suce-

Los autores participantes del trabajo declaran no tener conflictos de interés en relación con la empresa fabricante (Synthes ${ }^{\circledR}$ MatrixRIB, Solothurn, Suiza) y su distribuidora en Chile. No ha existido tampoco por parte ellos injerencia o financiamiento alguno en la preparación de este artículo, ni en la referencia de los enfermos.

* Servicio de Cirugía Torácica, Clínica Santa María, Santiago, Chile. 
de en el tórax volante y aunque exista evidencia publicada que recomiende la estabilización de las fracturas en estos casos ${ }^{3}$. De acuerdo a Mayberry y cols menos del $26 \%$ de una muestra de 405 cirujanos de trauma y cirujanos torácicos encuestados, han participado en una fijación quirúrgica de fracturas costales, existiendo desconocimiento en las indicaciones del procedimiento y gran variabilidad en las técnicas utilizadas para lograr la fijación ${ }^{4}$.

$\mathrm{Al}$ igual que en otros territorios, la reducción del foco de fractura mejora el dolor y restablece la funcionalidad. Sin embargo, dos factores hacen que en el caso costal esta tarea no sea trivial: el número y posición de las fracturas, y los dispositivos o técnicas quirúrgicas. Se han descrito diversas técnicas, como la sutura con alambre y puntos transcostales, los clavos intramedulares de Kirschner, las grapas, las placas de osteosíntesis (OTS) y las placas de material absorbible. Muchas de ellas carecen de material e instrumental dedicado específicamente a la reducción costal, o de estudios de biomecánica y de bioseguridad para esta indicación específica, que expliciten el riesgo de fatiga de material (fractura), desplazamiento y migración cavitaria, fractura a distancia por estrés, estabilidad a largo plazo y ausencia de dolor ${ }^{4-8}$.

En este artículo presentamos dos casos de reparación costal múltiple con OTS con placas y tornillos de titanio especialmente diseñados para su uso costal, y se discuten sus indicaciones, sus características técnicas y las ventajas clínicas observadas.

\section{Caso Clínico 1}

Hombre de 60 años de edad sin antecedentes mórbidos, ingresa 8 días después de haber sufrido una caída en motocicleta con golpe torácico lateral derecho. Las imágenes de ingreso demostraban un hemoneumotórax derecho moderado, fracturas costales derechas múltiples, 2 de ellas con fragmento intratorácico. El paciente refería intenso dolor (Escala Visual Análoga (EVA) 8 de 10) con imposibilidad de realizar sus actividades laborales habituales y pérdida de su autovalencia. $\mathrm{Al}$ examen físico la pared torácica se apreciaba inestable, muy sensible, con escasa excursión respiratoria y signos de ocupación pleural por líquidos. El estudio dirigido con tomografía computada de tórax (CT-tórax) demostró fracturas desplazadas de los arcos 8-9-10 derechos y no desplazadas de los 5-6-7 del mismo lado (Figura 1 a y b). Se realizó videotoracoscopía derecha, drenaje del hemoneumotórax y decorticación del lóbulo inferior. Los 3 arcos costales desplazados fueron reducidos a su posición anatómica y fijados con 3 placas de titanio derechas y tornillos bicorticales bloqueados, 4 por cada extremo (Synthes ${ }^{\circledR}$ MatrixRIB, Solothurn, Suiza) (Figura 2 a y b). La reducción de los focos de fractura costal (Figura 3) y su estabilidad final fue óptima, además no se requirió de la apertura del espacio intercostal. Su EVA fue 2 de 10 en el día 2 post operatorio, siendo capaz de tolerar la deambulación y la kinesiterapia respiratoria con analgesia oral.

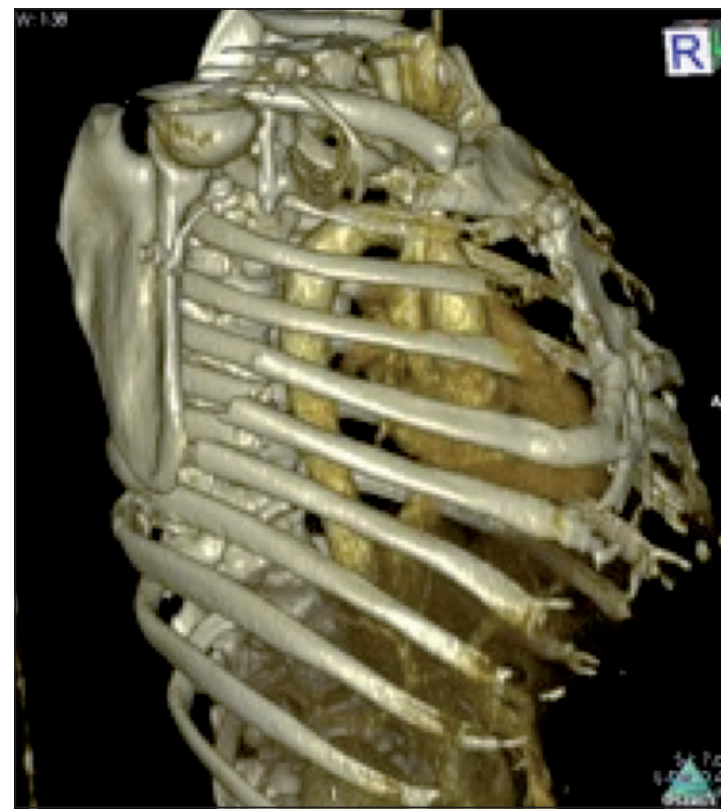

Figura 1 a. Caso 1, reconstrucción 3D de la parrilla costal de un paciente con fracturas costales derechas múltiples.

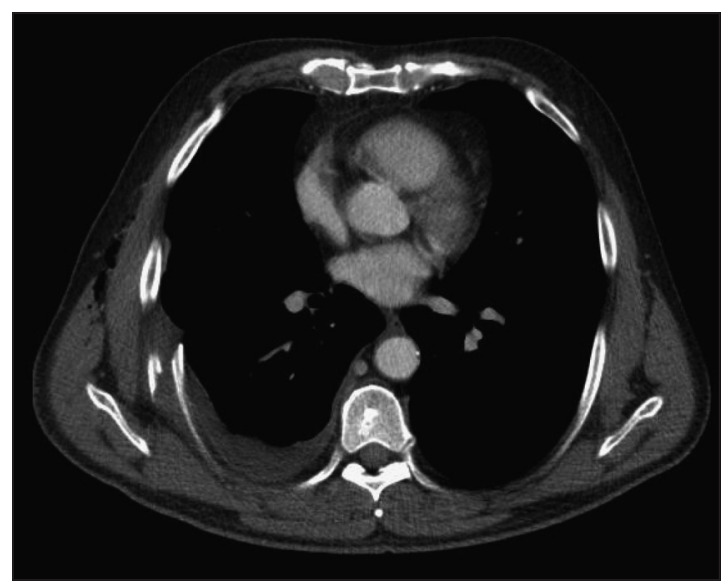

Figura 1 b. Caso 1, corte axial donde se aprecia un hemotórax derecho, y una fractura costal con fragmento intra pleural. 


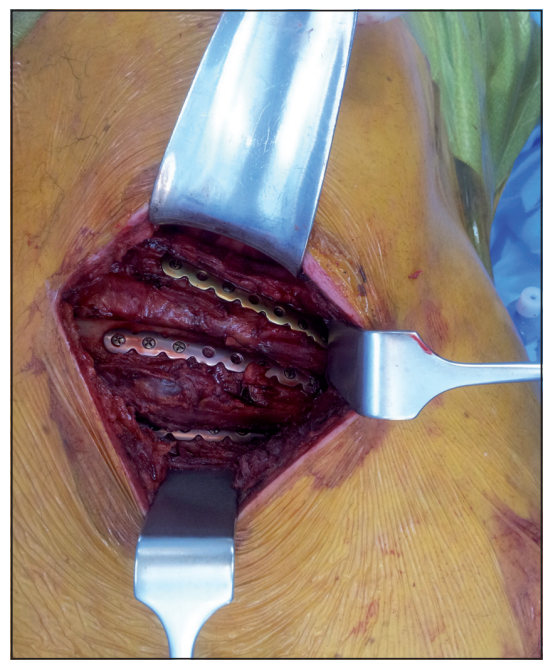

Figura 2 a. Caso 1, Osteosíntesis costal con tres placas de titanio y tonillos bicorticales bloqueados. Note la ausencia de toracotomía para realizar la OTS.

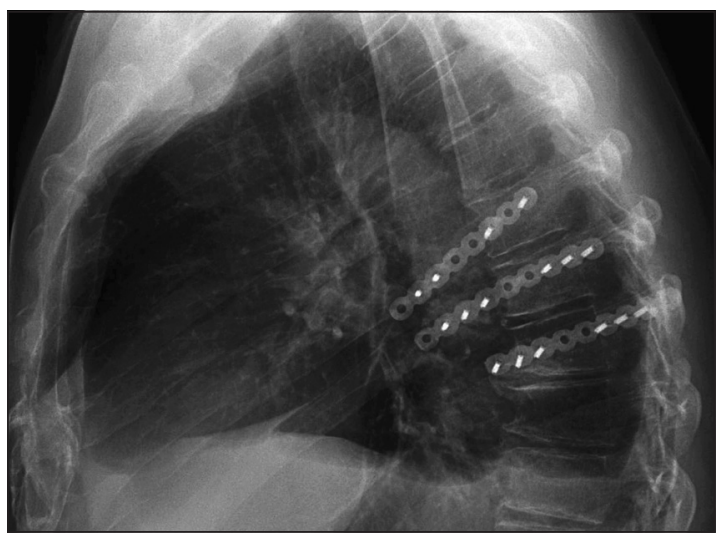

Figura 3. Caso 1, Control radiológico a los 60 días, sin signos de soltura o fractura.

\section{Caso Clínico 2}

Hombre de 60 años hipertenso y obeso mórbido $\left(\mathrm{IMC}=34 \mathrm{~kg} / \mathrm{m}^{2}\right)$, consulta por dolor crónico torácico lateral izquierdo bajo, secundario a traumatismo de pared acaecido un año atrás. En ese entonces, sufrió fracturas costales múltiples y evolucionó con falta de consolidación, fue operado, realizándose resección de un segmento costal izquierdo bajo más plastía con malla de polipropileno (Figura 4). Desde entonces presenta dolor local progresivo de tipo neurítico y de difícil manejo. Al examen físico destacaba una deformidad asimétrica de la pared torácica anterior con área indurada y disestésica en relación a la cicatriz de toracotomía, además presentaba flaccidez y protrusión de contenido tóraco-abdominal, lo cual se demostró con resonancia magnética de la zona.

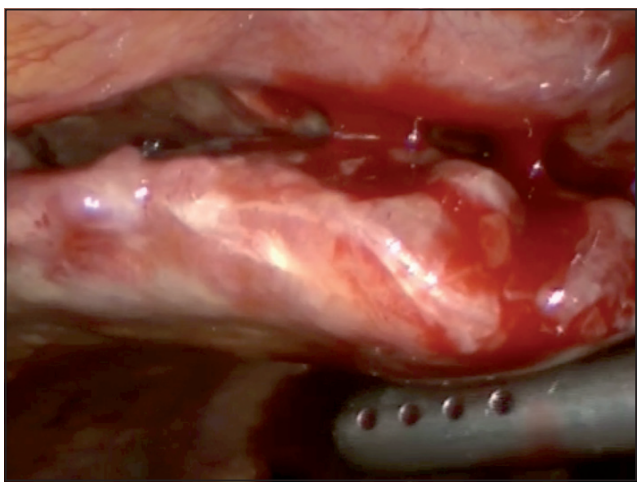

Figura 2 b. Caso 1, visión por videotoracoscopía de uno de los fragmentos conminuta intra pleural.

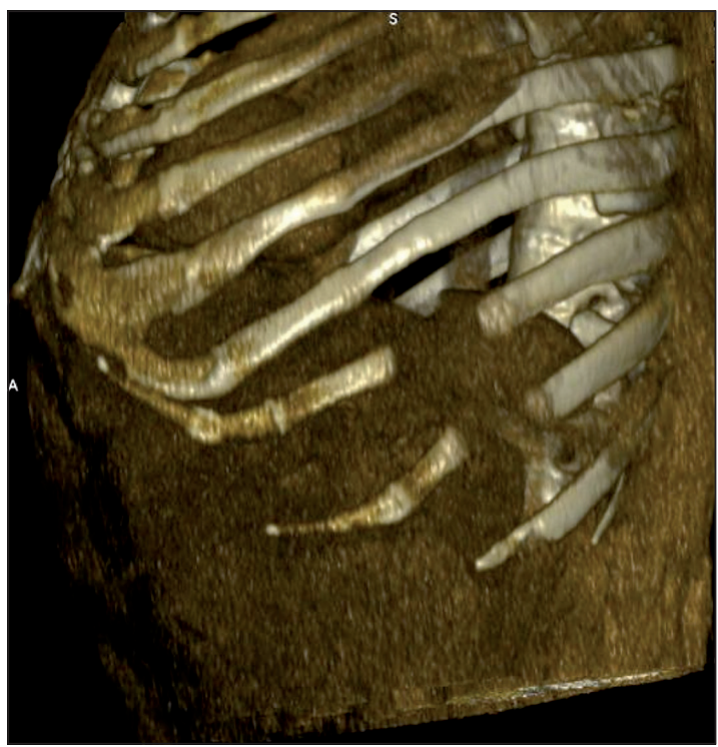

Figura 4. Caso 2, reconstrucción 3D de la parrilla costal, observe el defecto anterior e izquierdo.

Luego de un mes de entrenamiento respiratorio y muscular abdominal, se realizó toracotomía izquierda en conjunto con cirugía bariátrica (manga gástrica laparoscópica). Se encontró un gran proceso fibrótico en relación con la malla y un defecto parietal de 8 por $10 \mathrm{~cm}$. Posterior al retiro de la malla se realizó fijación de dos arcos costales con dos placas de titanio izquierdas y tornillos bicorticales bloqueados, 4 por cada extremo, logrando una reconstrucción óptima de los arcos costales (Figura 5 a). Finalmente se reparó el defecto parietal con Goretex (Dual Mesh®) fijándolo a los arcos costales (Figura 5 b), evitando el paquete vásculo-nervioso, y se realizó bloqueo con anestésicos y esteroides. El paciente evolucionó sin dolor importante, con EVA de 1-2 de 10 en los controles de los días 30 y 60 del período post-operatorio. 


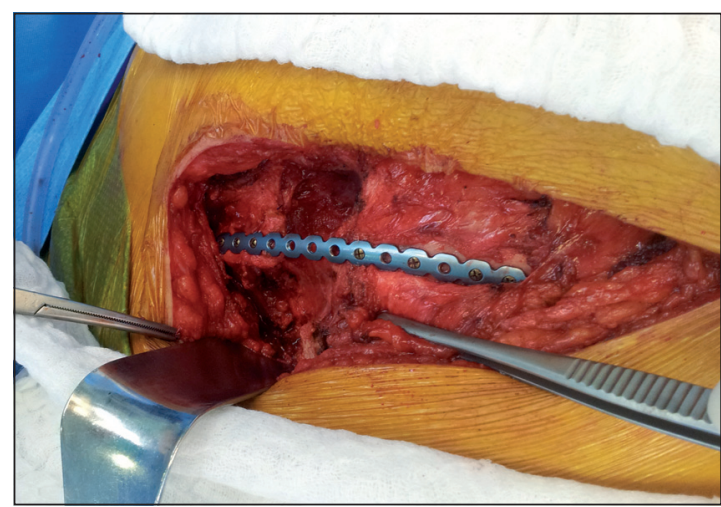

Figura 5 a. Caso 2, reparación de la pared costal con defecto intermedio utilizando un arco de titanio.

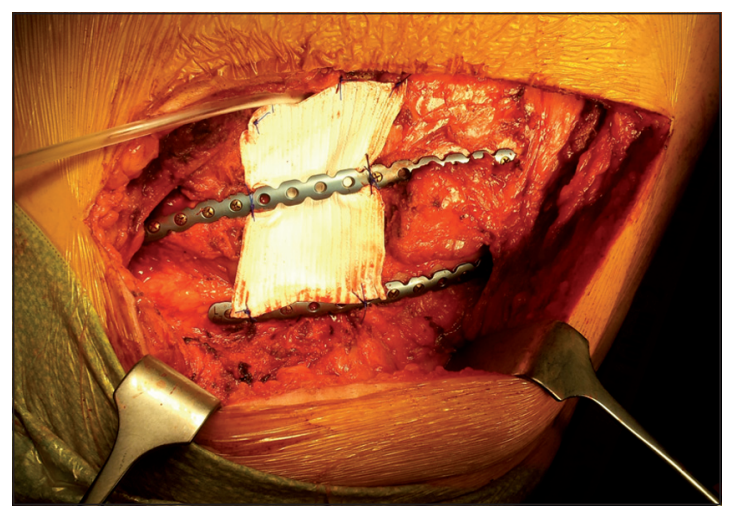

Figura 5 b. Caso 2, resultado final de la reparación. Se aprecia también la cobertura del defecto parietal con malla DualMesh ${ }^{\circledR}$ y dos arcos costales de titanio. Se utilizan al menos 3 tornillos por cabo, bloqueados y bicorticales.

\section{Discusión}

Las fracturas costales son lesiones traumáticas frecuentes, y habitualmente múltiples en el contexto hospitalario. El dolor es su síntoma central y el causante de morbilidad respiratoria importante, especialmente en adultos mayores y en pacientes con múltiples fracturas. También es posible atribuirle el tiempo que demora un individuo en retornar a sus actividades diarias usuales ${ }^{1,2}$.

La reducción ósea anatómica del foco de fractura costal restablece la funcionalidad (protección torácica y movimientos respiratorios) y disminuye el dolor y la impotencia funcional del segmento afectado. Sólo un pequeño número de situaciones se reconocen como indicaciones de fijación costal, aparentemente por su dificultad técnica, la falta de dispositivos diseñados específicamente para tal efecto, el desconocimiento de la biomecánica y compatibilidad de algunos dispositivos, y también por la falta de difusión de algunos estudios en este campo. Clásicamente se considera indicación de fijación quirúrgica el tórax volante, la presencia de fragmentos intra cavitarios, la impactación y laceración pulmonar, o la evidente deformidad estética; en muchas ocasiones condicionado a la necesidad de toracotomía por otra razón ${ }^{3,9-11}$.

La presencia de múltiples sitios de fracturas dificulta su acceso quirúrgico, y en muchas de las técnicas (puntos transcostales, fijación endomedular con clavo de Kirschner, grapas, puntos pericostales, etc.) obliga a realizar toracotomías múltiples con el consiguiente costo en dolor e injuria sobre la pared. La osteosintesis con placa no requiere de toracotomías múltiples (apertura del espacio intercostal y separación de él) ya que solo requiere de la exposición de su cara extra torácica para la fijación de la placa.

Un segundo elemento importante tiene que ver con las características de los dispositivos utilizados para estabilizar el foco de fractura. Los materiales idealmente deben ser biocompatibles, asegurar la reducción de la fractura para permitir la cicatrización ósea, restablecer las características mecánicas necesarias para la función costal (21 mil ciclos respiratorios por día) y minimizar los riesgos de soltura, fractura y/o migración. Actualmente existen placas absorbibles (no disponibles en nuestro medio), placas metálicas (acero o titanio) y placas-grapas (una mezcla de placa con aletas laterales para fijarse a modo de abrazadera no disponibles en nuestro medio), existiendo información biomecánica para dispositivos costales sólo en placas de titanio con tornillos bloqueados ${ }^{7}$, fijadores endomedular ${ }^{7}$ y placas absorbibles ${ }^{6}$. En particular la placa de titanio utilizada en este caso respeta la geometría del arco costal (convexidad), la rotación sobre su eje y la rigidez del arco (elasticidad), a diferencia de otras placas de OTS de uso maxilofacial $(2,4$; 2,7 ó 3,0 mm) que requieren ser contorneadas para lograr mayor superficie de aposición y que poseen mayor rigidez ${ }^{12}$. El diseño de la placa con bloqueo le entrega ventajas adicionales como la distribución uniforme de la tensión, inmoviliza mejor el foco de fractura para que ocurra la cicatrización, y permite la fijación de fragmentos conminuta $^{13}$. En placas absorbibles se ha demostrado que el estrés sobre los tornillos y la cortical varían con la cercanía al foco de fractura, con la movilidad costal y la fase de la respiración, explicando en algunos casos soltura de tornillos y de la placa. Es importante por tanto evaluar en el largo plazo estas características, hasta ahora escasamente notificadas ${ }^{14}$.

Las técnicas de fijación costal necesitan ser evaluadas en forma prospectiva y aleatoria versus 
el manejo analgésico conservador en un rango más amplio de indicaciones que el sólo tórax volante $^{10}$, e incluir en su evaluación de desenlaces, su costo-efectividad y las secuelas de largo plazo. Gran parte de la información clínica proviene de pequeñas series descriptivas prospectivas sin comparación contrafactual. La incidencia reportada de complicaciones es de 1 a $2 \%$ e incluye seromas e infección posterior a OTS con placas y tornillos.

En el primer caso la CT-Tórax y la videotoracoscopía permitieron identificar los arcos costales necesarios de reparar, con la ventaja adicional de realizar la exploración y el aseo torácico derecho sin requerir toracotomía. En el segundo caso, la evidente presión (expansiva) que ejerce en la zona de aposición del diafragma el contenido abdominal de un paciente con obesidad mórbida, nos hizo proponerle cirugía bariátrica como complemento a la reparación costal. La combinación de placas y tornillos de titanio más parche de Goretex (DualMesh ${ }^{\circledR}$ ) ha sido reportado como técnica de reparación de defectos torácicos mayores post quirúrgicos ${ }^{15}$.

Se muestran dos casos de reparación costal con placas de titanio y tornillos bicorticales bloqueados, especialmente diseñados para este efecto, con una muy buena evolución clínica post operatoria y en el mediano plazo. Como técnica quirúrgica ofrece múltiples ventajas en su acceso, implante y estabilidad del foco de fractura, restitución de la función costal y reinserción laboral. $\mathrm{Su}$ uso en indicaciones relativas dependerá de la evaluación prospectiva de la OTS costal versus terapias analgésicas no invasivas.

\section{Bibliografía}

1.- SIRMALI M, TÜRÜT H, TOPÇU S, GÜLHAN E, YAZICI U, KAYA S, et al. A comprehensive analysis of traumatic rib fractures: morbidity, mortality and management. Eur J Cardiothorac Surg 2003; 24: 133-8.

2.- KERR-VALENTIC M A, ARTHUR M, MULLINS R J, PEARSON T E, MAYBERRY J C. Rib fracture pain and disability: can we do better? J Trauma 2003; 4 (6): 1058-63; discussion 1063-64.

3.- NICE. Insertion of metal rib reinforcements to stabilise a flail chest wall [Internet]. NICE [date unknown];[cited
2012 Aug 30] Disponible en:http://www.nice.org.uk/

4.- ENGEL C, KRIEG J C, MADEY S M, LONG W B, BOTTLANG M. Operative chest wall fixation with osteosynthesis plates. J Trauma 2005; 58: 181-6.

5.- RICHARDSON J D, FRANKLIN G A, HEFFLEY S, SELIGSON D. Operative fixation of chest wall fractures: an underused procedure? Am Surg 2007; 73: 591-6; discussion 596-7.

6.- CAMPBELL N, RICHARDSON M, ANTIPPA P. Biomechanical testing of two devices for internal fixation of fractured ribs. J Trauma 2010; 68: 1234-8.

7.- BOTTLANG M, WALLESER S, NOLL M, HONOLD S, MADEY SM, FITZPATRICK D, et al Biomechanical rationale and evaluation of an implant system for rib fracture fixation. Eur J Trauma Emerg Surg 2010; 36: 417-26.

8.- CAMPBELL N, CONAGLEN P, MARTIN K, ANTIPPA P. Surgical stabilization of rib fractures using Inion OTPS wraps-techniques and quality of life follow-up. J Trauma 2009; 67: 596-601.

9.- NIRULA R, DÍAZ J J JR, TRUNKEY D D, MAYBERRY J C. Rib fracture repair: indications, technical issues, and future directions. World J Surg 2009; 33: 14-22.

10.- BOTTLANG M, LONG W B, PHELAN D, FIELDER D, MADEY S M. Surgical stabilization of flail chest injuries with MatrixRIB implants: A prospective observational study [Internet]. Injury 2012 Aug; disponible en: http://www.ncbi.nlm.nih.gov/pubmed/22910817.

11.- MAYBERRY J C, HAM L B, SCHIPPER P H, ELLIS T J, MULLINS R J. Surveyed opinion of American trauma, orthopedic, and thoracic surgeons on rib and sternal fracture repair. J Trauma 2009; 66: 875-9.

12.- LAFFERTY P M, ANAVIAN J, WILL R E, COLE P A. Operative treatment of chest wall injuries: indications, technique, and outcomes. J Bone Joint Surg Am 2011; 93: 97-110.

13.- EGOL K A, KUBIAK E N, FULKERSON E, KUMMER F J, KOVAL K J. Biomechanics of locked plates and screws. J Orthop Trauma 2004; 18: 488-93.

14.- MARASCO S F, SUTALO I D, BUI A V. Mode of failure of rib fixation with absorbable plates: a clinical and numerical modeling study. J Trauma 2010, 68:1225-33.

15.- BERTHET J-P, WIHLM J-M, CANAUD L, JOYEUX F, COSMA C, HIRECHE K, et al The combination of polytetrafluoroethylene mesh and titanium rib implants: an innovative process for reconstructing large full thickness chest wall defects. Eur J Cardiothorac Surg 2012; 42: 444-53.
Correspondencia a:

Dr. Mauricio Fica Delgado

Cirugía Torácica Clínica Santa María.

Teléfono Fijo: 27230593

E-mail: mfica@vtr.net - mfica@csm.cl 